\title{
The State of Astronomy Education in Iran: Challenges and Solutions
}

\author{
Saeed Jafari* \\ Saros Science Popularization Team, Tehran, Iran \\ Space Generation Advisory Council, Vienna, Austria
}

\begin{abstract}
Astronomy has a long history in Iran. Our ancestors were pioneered in astronomy and from ancient years was an excelled country in the construction and use of astronomical buildings. Today, there are more than 200 local astronomical societies and centers in the country that the amateur astronomy community has played a significant and influential role in the education and promotion of astronomy to the general public since 2000. A greater part of astronomy education was provided by amateur astronomers through outreaching events, observing nights, and education and outreach projects. Another part of it has been disseminated by science journalists who are amateur astronomers and astronomy teachers in the media and newspapers. Considering the enormous potential that has existed in the growth and development of Iranians in the field of astronomy, in the meantime, challenges such as the specific cultural and social constraints for women's activities, lack of sufficient knowledge of the teachers as well as the training required for them, the lack of a standard curriculum for teaching astronomy and network of astronomy teachers, and also economic barriers, have caused socio-economic development and education through Astronomy to grow less in Iran. This survey suggests challenges for teaching astronomy in general.
\end{abstract}

Keywords: Astronomy education, Education and public outreach, Amateur astronomy, Innovation in astronomy communication, Scientific literacy, Iran

\section{Introduction}

The first moves in the formation of informal and amateur astronomy in Iran date back to the 1960s and the first step in this direction was taken by the Institute for the Intellectual Development of Children and Young Adults in Tehran. Thus, a planetarium was set up in one of the centers of the public library in the district of Shohada Square which attracted and engaged the youth and general public of the city. After the Islamic Revolution (1979), the Office of Research and Planning of Textbooks affiliated with the Organization for Educational Research and Planning was formed, but there was still no independent textbook for teaching astronomy among dozens of textbook titles, until in 1997 a new and independent book called "An Introduction to Astronomy" was written by Dr. Mohamadreza Heidari, Dr. Mohamadreza Khajepour and Dr. Mohammad Taghi Mirtorabi and entered the education cycle.

Each year, education and outreach in Iran create over 800 astronomy engagement opportunities for the general public and astronomy enthusiasts, but to make all of this possible, educational programs and outreach need the cooperation and support of numerous teachers and outreach professionals volunteers and relevant educational institutions as well.

The needs of tomorrow's society required us to be able to educate our children today and live in the future. Being satisfied with what we have today is to ignore the future. If nothing happened in our lifetime, we needed again to think about tomorrow. While the roles and needs of individuals in the present age are changing. Thinkers emphasize that education is one of the most important

*sjafari@saros.ir, saeed.jafari@spacegeneration.org 
infrastructural institutions that can fulfill this important goal and prepare people for tomorrow's life. Producing the proper and innovative educational materials is one of the most important tasks of education in achieving this goal.

This article attempts to describe and suggest some challenges facing astronomy, mainly in education, but also relating it to a wider social setting. The paper goes on to suggest a series of underlying reasons for why we should consider these challenges and solutions for our current situation in astronomical society in Iran. This description is tentative and explorative, and is meant to give ideas for a discussion of possible explanations.

\section{How astronomy teachers can make the most of their effective par- ticipation}

\subsection{Schools need qualified teachers in astronomy and space}

Teachers can change the world. We must have skilled and experienced teachers with sufficient knowledge of teaching that arena to inspire students. In Iran, teaching astronomy classified into three different societies. The first society is academic society which working on professional topics in astronomy and physics in university level. Second, society is amateur astronomy community that teaching amateur astronomy by education and outreach programs and in some institutes and NGOs by some private and public courses on different levels. Third, the community of teachers who teaches elementary concepts of astronomy, which are part of the content of natural science, geography, and physics textbooks in school. Teachers society is the most effective and influential group that has the ability to get teens interested in astronomy (in early, middle and late adolescence) in primary stages of education and learn them the scientific concepts in an accurate and precise way. Furthermore, amateur and professional astronomers are among the most influential people who have been teaching amateur astronomy to the general public through scientific experiments, observing nights in schools and universities and astronomy outreach activities over the past two and three decades in an environment outside the classroom of students and sometimes even inside schools. The basic question is how many teachers are qualified for teaching astronomy in schools or outside of the educational environment? Are the learning resources and materials of students and learners prepared and presented according to their age range? What are the teaching methods and principles for each age group and how do they develop it? Answering these questions is a remarkable issue that should be considered by every teacher, especially those who are teaching in schools, and teachers who have been continuously organizing astronomy courses at private institutions and science-based organizations for many years. Acquiring knowledge of a science and how to acquire the teaching skills of that field to the learner are two different scopes that every teacher should be aware of and pre-study before starting teaching.

\subsection{Astronomy curriculum is a key factor to sustain and develop the interest in astronomy and science}

Curriculum development is not an easy task to perform or the responsibility cannot be given to anyone out of the blue. Idea implementation and its related policies are somewhat different in each country based on the social, cultural, political and religious factors, of course, if it can be implemented in the best possible form and shape. Implementing the astronomy curriculum known as "The universe, earth and life" during primary and high school education, works as a course that can engage the minds of students at any time instant by considering the learners' perception of the world around them and teach them a different look at life and the complex world around them; in this way, their knowledge of the world around them and the place they live in, as well as their responsibility as an amateur human being on the planet earth, increases after graduating from the school. 


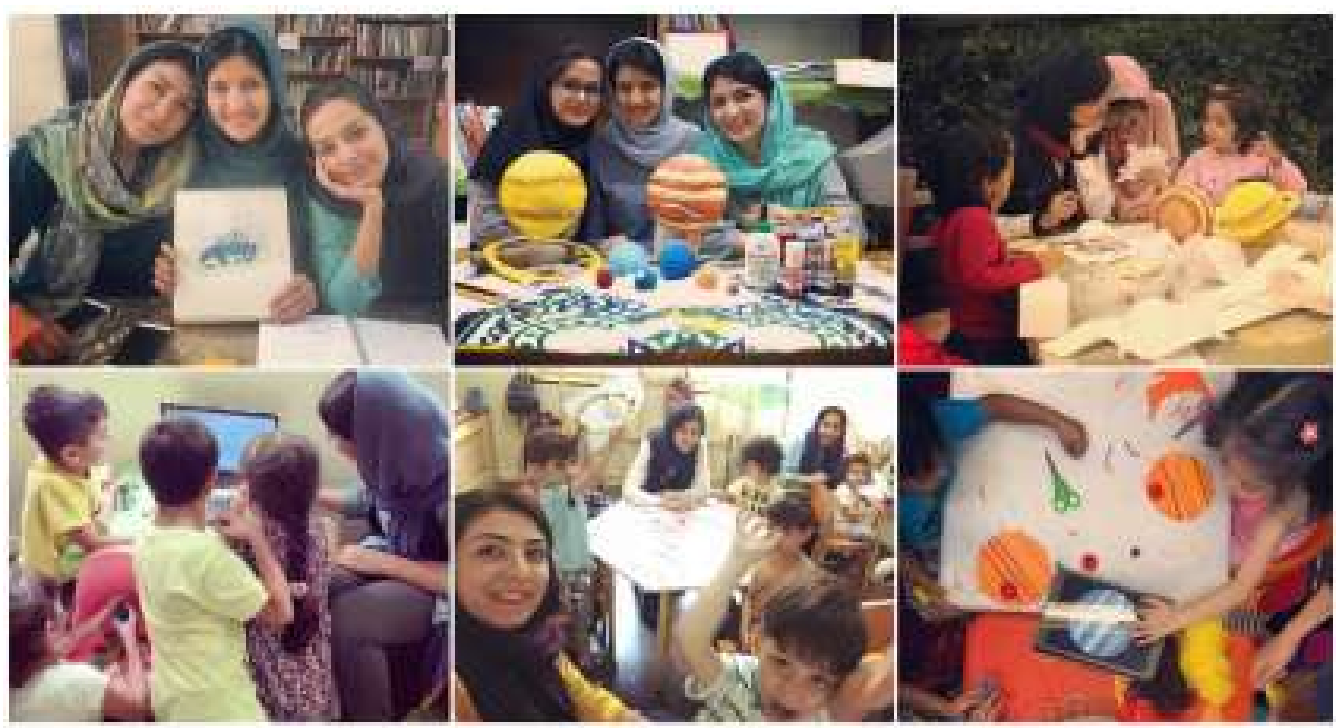

Figure 1. Andromeda is a mobile educational team with the aim of promoting science and astronomy and sharing the joy of learning science with all interested parties, especially for children and youth.

\section{How to implement and evaluate astronomy education in outreach activities and programs}

\subsection{Who needs astronomy - and why?}

Sharing knowledge with a group of students who don't have any particular interest in science, or politicians who do not pay much attention to topics and challenges of modern science, or even answering the challenging questions of individuals who believe in fortunetelling and astrology more than astronomy, i.e. people who have a guard against the word "scientific", could change their point of view regarding this branch of science. How to engage and involve minds of individuals from different groups is a vital and fundamental issue that some astronomy communicators and teachers are unaware of. First of all, before trying to engage the people of a society with scientific issues or answer their questions, we should ask ourselves questions such as what benefits and advantages would knowing this science bring for them? How does this science help improve our lives? What is the necessity of knowing and promoting astronomy in a situation where more than 736 million people live in extreme poverty? What is the importance of science and technology in education? What is the importance of knowing astronomy and sending probes into space in today's society? Along with pseudoscientific-like defensive answers and questions that always grow in the turbulent path of astronomy teachers and communicators. The answers to these questions make it clear to us that those who want to (or have) become interested in this science in the first point of contact, should not limit astronomy merely to the beautiful images of nebulae, or the discovery of a galaxy at the farthest point in the universe. The nature of this science is inclined to find out who we are and what is our place in the universe. Rather than describing astronomy to anyone, we have to convince ourselves regarding nature, why and how and the uses and applications of this science from all aspects, so that we can make the importance and necessity of knowing it in the face of challenging questions from people and lay audience, more understandable.

\subsection{Choosing the target audience and how to engage and educate them}

What topics should say to who person? Educational topics that offered for teenagers are different from topics that offered for adults. The percepts of audiences are different by noticing to some relative factors such as education, social situation, culture and literacy for understanding social complexity of different topics. identifying of these factors are change in different situations. For example, if you are in the class with teenage students you can extract personal features about that age group. But if you 
are in the conference which the audience of that is all group of people who are interested in astronomy, before beginning your offered you faced by some statistical questions and you should ask the audiences to answer the questions by raising their hands. Or people should ask the lecturers that ask some short questions by the form of question and answer to the participants in middling or beginning of their offered. So we can receive effective group information from program audience level by noticing to the items of breaking time, networking, chatting and having reflection and challenge questions. Generally, teachers and communicators of astronomy should notice to this part that for presenting astronomy content to different audiences, introducing surprising facts which tells for the public is not necessary. Sometimes by noticing to the time and the need of that group we can get them some information and distinct story tellings.

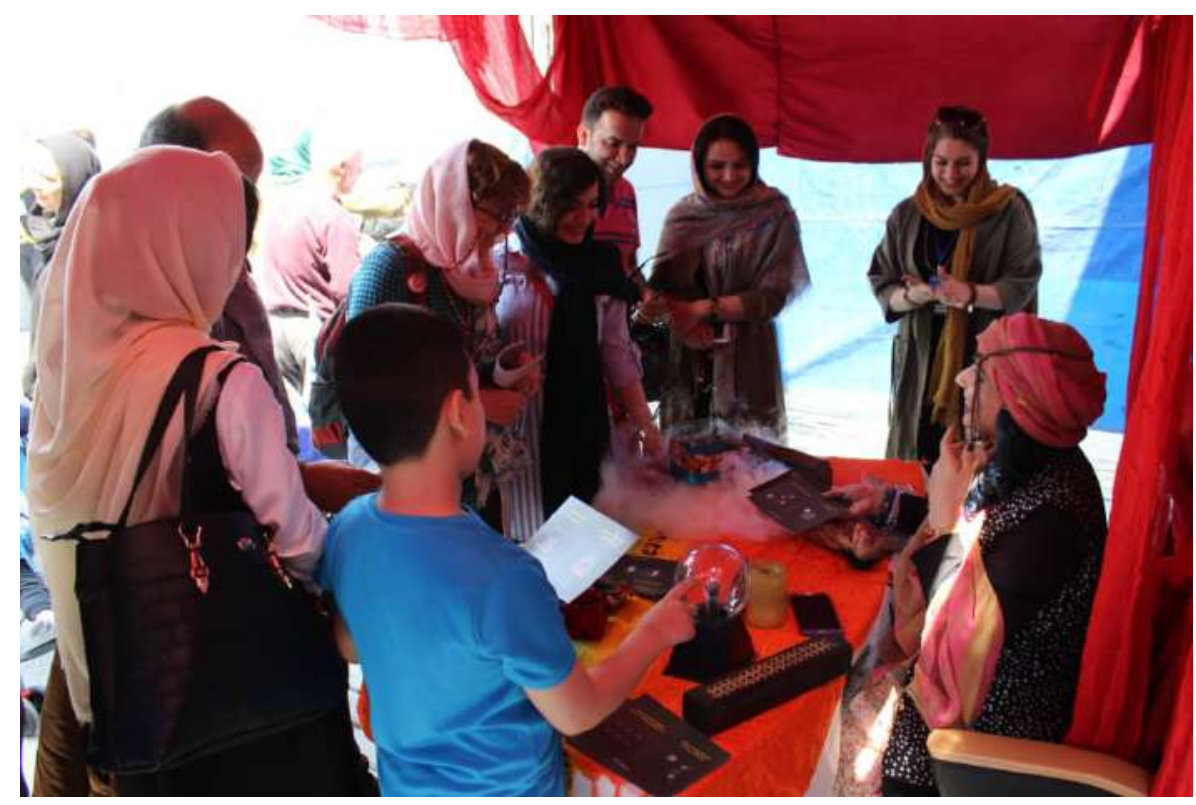

Figure 2. An amateur astronomer and graduate student of philosophy of science who plays the role of an astrologer to discusses challenging questions about how much the different groups of people believe in astrology and superstition.

\subsection{The need to organize astronomy teacher training program and create a net- work of astronomy teachers nationwide}

The presence of an excellent teacher is important for students' progress, therefore, it is of incredible importance that teachers are not just told what to teach but how to teach. Before they enter the classroom, and throughout their time in the classroom, we must focus on their own learning and development as much as we focus on pupils. As mentioned earlier, teaching astronomy in Iran is pursued in a limited range only in a few schools and mostly in out-of-school in scientific and astronomical institutions, centers and groups. In addition, the community of amateur astronomers has played a vital role in teaching amateur astronomy to the public in scientific and cultural environments. Given the linguistic, cultural, and geographical diversity in Iran, half of the astronomy teachers spectrum, have to consider the geographical and linguistic considerations when teaching astronomy to their corresponding learners, and certainly each of them has had a different experience in their own cities and villages, compared to the teachers living in metropolitan areas. The explanation of astronomy and physics topics and notions, and their perception by the learners are somewhat different based on the language and culture of the area they live in. Therefore, it is necessary to use these experiences, teaching challenges, and up to date educational methods in a conversation hall in order to increase the knowledge of teachers. Holding annual events for training the astronomy teachers, is a way for gathering the educators in this field, so that they can both increase their knowledge in the specialized areas of education, psychology, and methodology, and update themselves on various astronomy topics in the world and their educational and training aspects. 


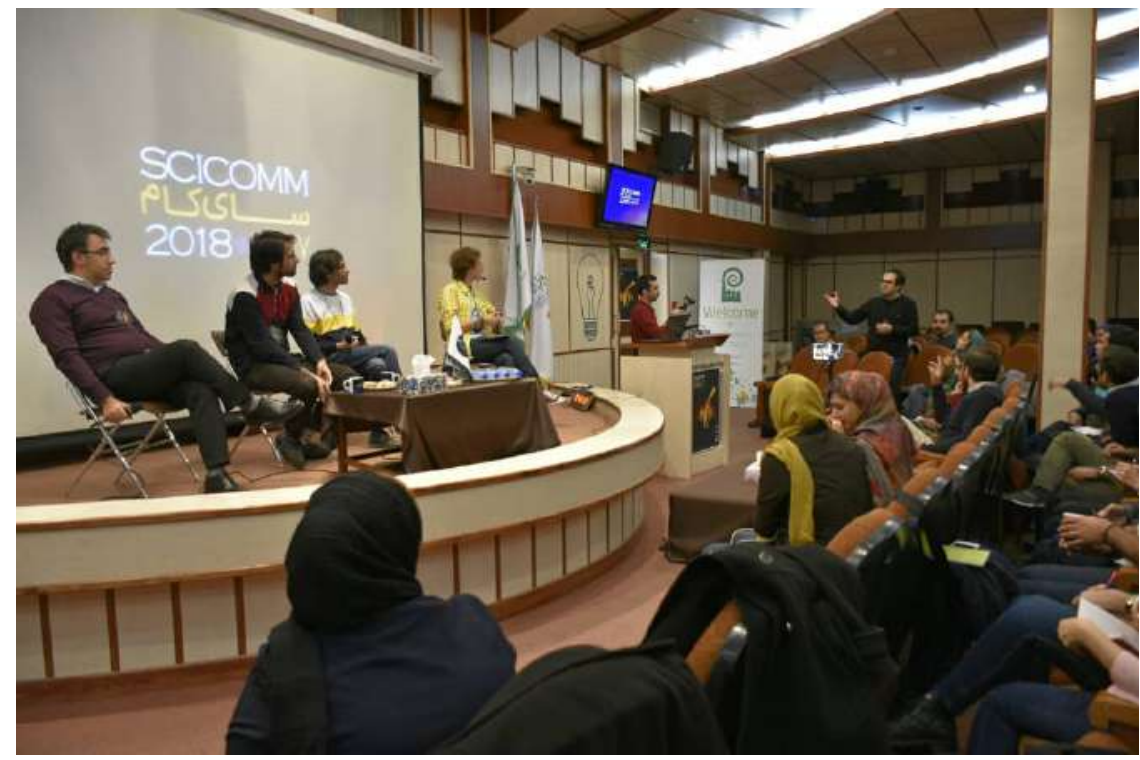

Figure 3. The first SciComm Conference brought together science communicators, amateur astronomers, scientists and science journalists and to give talks that are challengeable and solutionfocused.

\subsection{Creating a Farsi language hub to provide tools, programs and resources in astronomy education for organizers, teachers and parents and academia}

Astronomy teachers require contents and resources to be able to educate their students in different educational stages, both theoretically and practically. The scope and categorization of these resources could be such that make them applicable in out-of-school educational environments and in outreach activities. Currently, the majority of educational resources and tools are provided freely, and mostly in English, to the public by well-known space agencies and observatories and also the largest relevant scientific/astronomical institutions. Some of these open educational resources are designed based on the educational and cultural systems in other countries; although such resources can still be implementable in every country, design and production of such resources that are formulated based on the national considerations of each country and are based on the country's culture and society, could help the teachers, students, and their parents to have access to these resources and educational documents in their native language and via translated instructions. In addition, the scientific, historical, and cultural achievements and legacies of each country are the most important and valuable information that each country should be diligent in preserving and teaching them to the general public. Thus, the method of preserving this heritage in the education curriculum of schools and holding camps to visit historical, natural, and cultural heritage of the country would help in gaining a better understanding and learning of the topics.

\section{Astronomy education in media and society - current challenges and possible solutions}

\subsection{Gender Equality issues in astronomy: a snapshot of real and/or perceived causes for the gender gap}

A considerable part of the amateur astronomers in Iran is comprised of women, and they have the greatest participation in teaching astronomy in and out of schools, especially to children and teenagers. A significant number of these women are currently undergraduate or graduate students in the fields of physics and astronomy, however their incomes as a teacher are so low that even the men who have long been interested in teaching astronomy in and out of schools, choose this profession only temporarily, and are not able to accept this profession as their main job due to its low income. On the other hand, girls are forced to be content with this low income, because the lack of a satisfactory 
job corresponding to their field of study, does not allow them to easily find and work in any job. Over the past two or three decades, girls who were born in religious and traditional families, were not easily able to obtain the permission of their parents in writing in order to attend out-of-time observing programs and tours at night, or even attend and teach in these gatherings as an expert. However, these limitations have been largely solved among some families. The Sepideh's documentary tells the story of some Iranian girls who get into problems with their families regarding their interest in astronomy. Poverty, geographical isolation, minority status, disability, early marriage and pregnancy, gender-based violence, and traditional attitudes about the status and role of women, are among the many obstacles that stand in the way of women and girls fully exercising their right to participate in, complete and benefit from education (UNESCO).

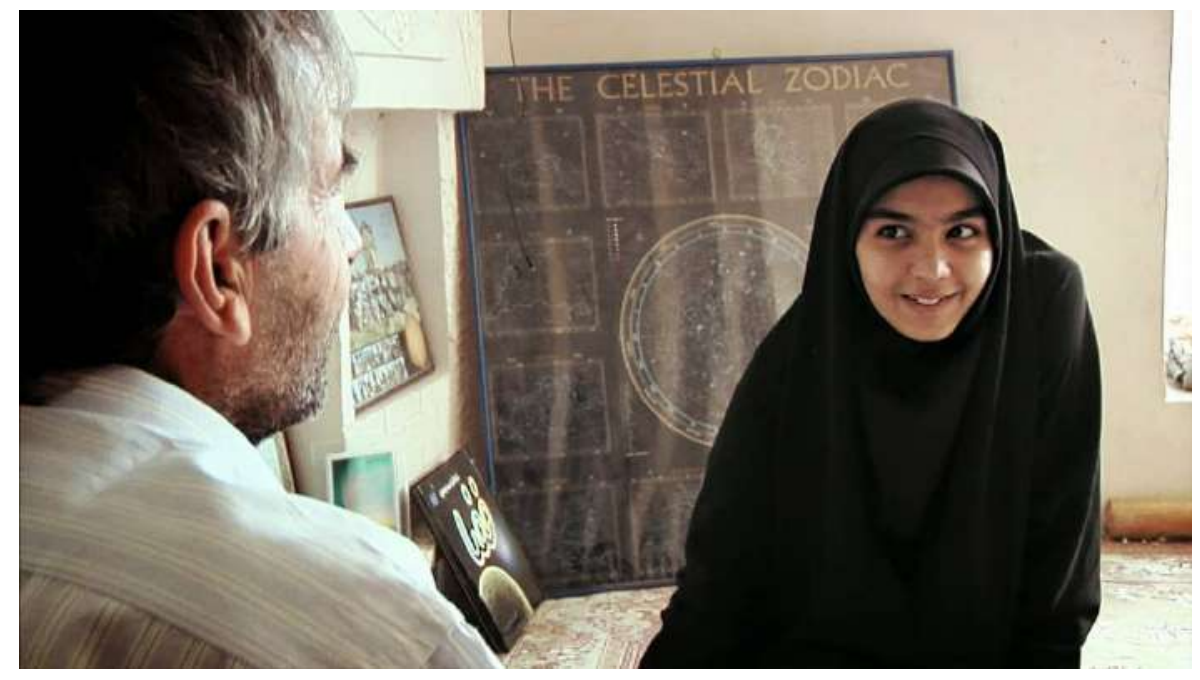

Figure 4. A scene from the Sepideh: Reaching for the Stars documentary and Sepideh's physics teacher, Mr. Kabiri, the man who started the astronomy club in Saadat Shahr city.

\subsection{Combining astronomy and entrepreneurship can lead to new practice oppor- tunities in innovative education and outreach projects}

The consumer basket of any science, is complemented by products, resources and programs that provide a combination of the science and the product for fans, enthusiasts, and public. This product could be either an educational content presented to the user through an application, or a product in the shape of intellectual games and educational and entertaining goods. A great deal of astronomy enthusiasts from different fields of study and the astronomy experts, have ideas in mind, that not only help the events, classes and different scientific activities as a product, but also could be commercialized in the form of entrepreneurial ideas, and used to gain money both online and face-to-face. These activities in culture, art, media, and tourism, or any other field that can connect astronomy with entrepreneurship, could advertise and even promote astronomy in the form of a consumer product, and gradually lead to socio-economic growth of this science among other fields of economy. Today, some grassroots scientific organizations, centers, and groups, have gained the opportunity to better present their products and digital media advertisements and public relations and communications services in cooperation with these business and grassroots entrepreneur groups.

\subsection{Teaching and bring up amateur astronomers and astronomy educators to learn science journalism as an essential skill or alternative profession}

Nowadays knowing journalistic knowledge in the online environment is essential for those who are producing multimedia science content in the online world to keep people informed of science and technology news. Science journalism is one of the fields along with science communication which has been promoting science for many years and by asking about the accuracy of scientific topics, especially in basic and natural sciences, from experts and scientists to restates the knowledge of that field in 
simple and fluent language for the general public and lay audience in print and online media. Learning science journalism as a skill that can cover astronomy and space news in a more accurate and fluent way with the appropriate publication method in print and online media for active amateur astronomers which is the main arm for organizing astronomy education and outreach programs in the online and face-to-face environment in Iran is significantly important. In addition to strengthening and developing the knowledge and skills of narrating, reporting, authenticity and news analysis allows the astronomy community to make the most impact on education and scientific literacy among the general public through a variety of intellectual styles. Moreover, improving and developing the knowledge and skills of narrating, reporting, authenticity, and news analysis allow the astronomy society to make the most impact on education and scientific literacy among the general public through a variety of intellectual styles, besides, when pseudoscience and superstition are spread, and become popular through a viral process of Internet sharing, they will be able to use their journalistic ability and writing power to enhance general public understanding and awareness of fake news on scientific websites and wellknown news agencies. Moreover, the implementation of this certainly applies to the production of digital video, visual and audio content, which today are among the most visited and influential places to change people's viewpoints and perspectives on scientific topics and news.

\subsection{The need to learn and design innovative ideas for teaching and learning as- tronomy on different platforms}

In recent decade by developing technology and availability of free resources, materials and training tools in online spaces, besides production and implementing ideas for attracting all kinds of people through face to face outreach activities has led to a significant increase in the volume of content and the number of education and outreach programs and projects, especially in the social media atmosphere. In the meantime, the implementation of repetitive and common ideas in these spaces is less effective in engaging astronomy enthusiasts and the general part of people in different age groups. Today, innovative, creative and original ideas should be used in astronomy education and outreach programs in order to influence and dignity of the content on the audience's mind as much as possible, otherwise, astronomical content is duplicated and monotonous for the audience. This is why the general public does not follow remarkably the contents of astronomical groups in the midst of countless content, or only follows some groups that have popular ideas and new projects in the field of teaching and promoting of astronomy. The tastes and styles of the audience are varied in the online environment and the type of interest they have in joining these spaces is also different. Learning and produce videocast, podcast, infographics, application and software, scientific blog in form of web series are among the tools that help to better engage and captivate the audience and the importance of news and astronomical topics for its followers. Besides, astronomy can be brought astronomy to society by giving public lectures to the public in an organized program through various local, national and international events that need to communicate with the audience and engage them face to face with astronomers, scientists and communicators and using different gates to gather the public such as booth performance design, holding a conference, educational workshop and exhibition interest them to the world of astronomy. Among different multimedia project, String Cast podcast, is an example of success and popular digital audio file that attracting a lot of people to science narratives such as astronomy and space topics by using narrative ability and the storytelling structure of its creators with the theme of "strange corners of science". They have nailed their audience into these narratives wherever they can hear it. In the part of projects which are trying to attract and bring astronomy among people, there is a mobile observatory known as Tara Observatory. This particular idea plus abundant competency and perseverance was able to solve the problems of observatories which are usually located in the outside cities and far from living parts to run moving anywhere and make it a unique opportunity for people to easily observe the sky. This is certainly a nonstop adventure. This observatory also can move to the furthest regions of Iran and introduce people to the science of astronomy and the beauties of the night sky under the dark skies of cities and villages far and near. 


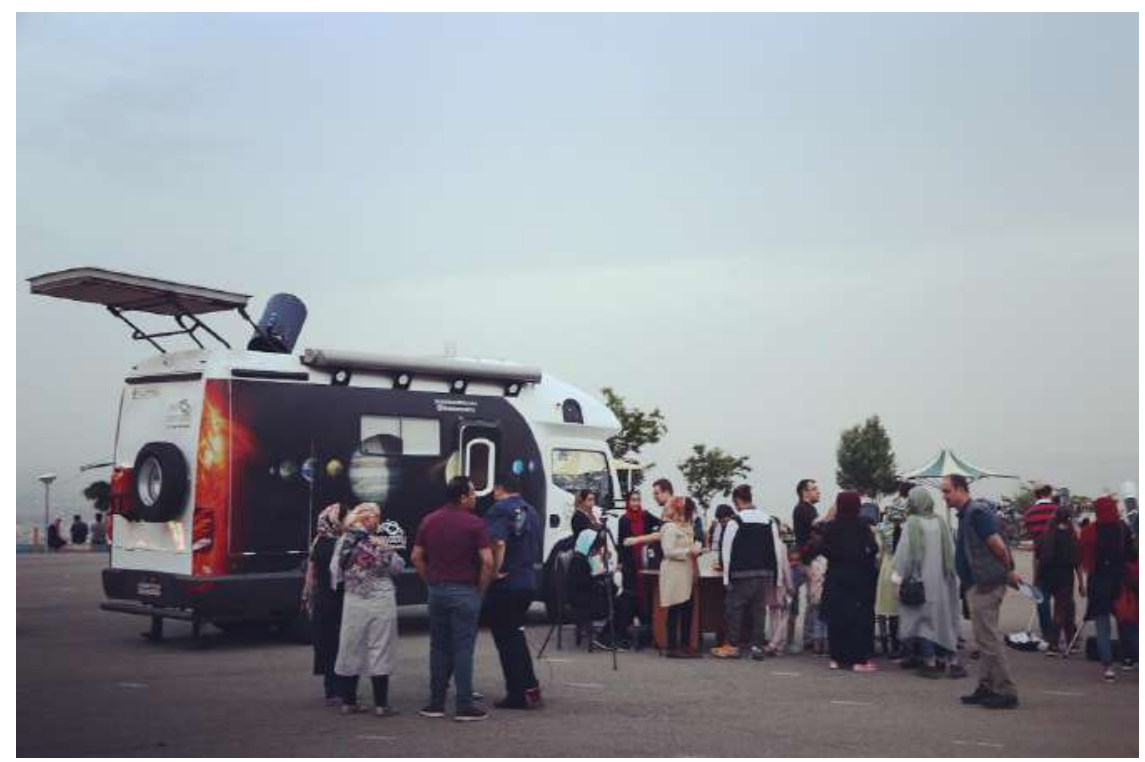

Figure 5. The Tara observatory is home to one of the largest telescopes in the country which allows the general public to observe the sky by entering the van.

\section{Conclusion}

However, it seems that astronomy, in terms of its unique features has easily attracted everyone's attention and interest. Therefore, it can be said that its development has been more successful by providing its teaching tools and resources in an amateur way (outside of school and textbooks) in Iran. The same feature of astronomy is observed in other countries.

We should promote the integration of creativity and design into education curricula to drive innovation, as well as incorporating contemporary socio-economic and educational issues into higher education studies and science communication research to foster progress in astronomy education and outreach in Iran. The results of these include the role of astronomy in capturing the public's attention and thereby promoting general science literacy and proficiency, its service as a gateway to science, technology, engineering, and mathematics careers. 